\title{
THE RELATIONSHIP BETWEEN SENSATION SEEKING AND SPEED CHOICE IN ROAD ENVIRONMENTS WITH DIFFERENT LEVELS OF RISK
}

\author{
Tyron Louw, Foroogh Hajiseyedjavadi, Hamish Jamson, Richard Romano, \\ Erwin Boer, Natasha Merat \\ Institute for Transport Studies, University of Leeds, Leeds, UK \\ Email: t.1.louw@leeds.ac.uk
}

\begin{abstract}
Summary: This paper presents the results of a driving simulator study conducted for the UK-funded HumanDrive project, which aims to develop natural, humanlike autonomous vehicle control. As part of that effort, this paper examines whether the established relationship between different sensation seeking (SS) traits and speed choice holds true across a range of driving scenarios, with different levels of contextual risk. Risk was introduced by varying a number of factors, including the environment (rural/urban), and the road edge context (low risk, static risk, potentially dynamic risk). Correlation analysis was performed between sensation seeking and the $95^{\text {th }}$ percentile of vehicle speed for roads with different levels of risk, also considering age and gender. The results indicated that, overall, SS was significantly positively correlated with the $95^{\text {th }}$ percentile of vehicle speed, and particularly for drivers under 40 years. SS was also found to correlate positively with speed choice at all risk levels, however, the effect was more pronounced in road environments that were classified as less risky. These findings have design implications for the development of autonomous vehicle control models.
\end{abstract}

\section{INTRODUCTION}

This paper presents the results of a study conducted for the UK-funded HumanDrive project, the ultimate objective of which is to develop natural, human-like controls for automated vehicles (AVs), so that their use, acceptance and trust by occupants, and other road users, is enhanced. The results presented here are linked to an experiment that exposed drivers to a set of repeatable driving conditions, where road and environmental factors were used to change the level of driving demand, and varied to shape the perception of the driving environment. The aim was to explore how speed choice and lateral position within the lane are affected by roadside furniture and oncoming cars. The main aim of the project is to use data from such definable behaviors to generate driver models that will quantify individual driving styles (see also Hajiseyedjavadi et al., 2019). The secondary aim of this study, and the focus of this paper, in particular, was to examine whether there is a link between these individual driving styles and different personality traits, and whether this is linked to the demands of different driving environments.

From a human factors perspective, there are currently no conventional or universal definitions and guidelines regarding what is good, safe, comfortable or acceptable behavior exhibited by an $\mathrm{AV}$. At present, navigation and performance by such vehicles are achieved via the use of environmental and infrastructure-based cues, such as road geometrical features, central and edge lane boundaries, and posted speed limits, for navigation, with sensors and radars used for obstacle detection and avoidance. However, while much effort has been invested in the engineering of such vehicles, it is currently not clear how this (ideally) flawless, machine-like, behavior is perceived by human drivers, whether the same behavior is desired by different user 
groups, and whether acceptance and uptake of AVs is affected by other human factors such as how social norms of the area affect driver behavior. Knowledge on whether humans prefer a more human-like AV is currently lacking, although research from other domains, such as studies on human-robot interactions, suggest that robots with humanlike design cues may have a positive impact on acceptance (Duffy, 2003). The opposite may negatively affect the trust, acceptance and use of these systems, and, in turn, adversely affect the potential benefit that they may bring to improving road safety (Hartwich, Beggiato, \& Krems, 2018). In addition, drivers are not homogenous, and differ in their driving styles, personality traits, risk-taking behavior, and hazard perception (Deery \& Fildes, 1999), as well as in their conception of a safe or expert driver (Mirman \& Kay, 2012). Risky driving behavior emerges when there is a mismatch between a driver's ability to manage their adopted safety margins given the driving demand imposed by the environment. The three-way dependency between attention, safety margins and demand manifest in different ways making it difficult to simply state behavior is risky when a safety margin is small or a driver is distracted. Driving demand refers to the frequency, magnitude and likelihood of perturbations that the driving environment imposes upon the driver such as sharp curves, lead car braking, side incursions, etc. For a given driving demand, drivers can tradeoff safety margins and attention; for example, by slowing down, adopting a longer following distance or shifting away from potential danger in a lane the driver can pay less attention. Therefore, it is not necessarily as risky for an attentive, skilled driver familiar with the road to drive faster and closer to the road edge, compared to an inattentive, novice driver unfamiliar with the road who exhibits the same behavior; this is especially true when the threat of unexpected dynamic elements is low. It is expected that individual differences in behavior are partly due to skill but also to the risk they attribute to possible unexpected or less predictable events, which is where we expect risk seeking and locus of control to manifest. What is currently not clear is whether different types of behavior from AVs are desired by different driver groups and personality types.

One trait which is shown to be well-correlated with risky driving behaviors, such as speeding, and risk of collisions (see Jonah, 1997, for a review), is sensation-seeking (SS, see Arnett, 1994), For example, in a simulator study examining behavioral adaptation to adaptive cruise control (ACC), drivers with high SS scores were more likely to engage in a demanding secondary task when their vehicle speed and headway was controlled by ACC (Rudin-Brown and Parker, 2004). Gianfranchi et al. (2017) showed that both high SS, and bad decision-making (as measured by performance on the Iowa Gambling Task), contributed to poor or risky driving performance. However, most studies in this context have relied on self-reported questionnaires for recording violations in driving. Also, while multiple studies have shown that the road context is a significant contributing factor to risky driving and crash severity (World Health Organization, 2005), few have explicitly examined how varying road and environmental risk factors affect behavior of different user groups, with different personality traits. It can be argued that by conforming to the rules of the road, maintaining the posted speed limit, and adhering to the central path of a lane, AVs are akin to low sensation-seeking, low risk-taking drivers. Therefore, in order to achieve the ultimate aim of the HumanDrive project, it is important to understand how different road contexts, portraying different types of risk, are managed by varying driver groups, in order to build controllers which are more representative of a wider range of drivers. As a step towards this aim, the current study provides an overview of the driving style and risktaking propensity of a group of drivers varying in age and sensation-seeking propensity, to establish the relationship between driving behavior and personality trait across a rich database of road environments present in UK roads. 


\section{METHODOLOGY}

\section{Participants}

Following ethical approval (LTTRAN-086), forty-four drivers were recruited, using the University of Leeds Driving Simulator database. Using crashTable 1. Summary of participant demographic information based statistics (Loughran \& Seabury, 2007) it was hypothesized that driving style and risk propensity may be influenced by age and experience. Therefore, drivers were recruited from four different age groups to gather data from a cross-section of the driving population, with age acting as a proxy for experience. Due to its challenging environment, participant dropout rate (mostly due to simulator-based motion sickness) was higher than anticipated, especially for the older age group. Table 1 shows the demographic summary of the final 35 participants who completed the study.

\section{Apparatus}

The high-fidelity University of Leeds Driving Simulator (UoLDS) was used in this study, which is based around a 2006 Jaguar S-type, housed within a $4 \mathrm{~m}$ diameter, spherical projection dome, with eight visual channels, rendered at $60 \mathrm{~Hz}$, at a resolution of $1920 \times 1200$, providing a horizontal field of view of $270^{\circ}$. The simulator also incorporates an eight degree-of-freedom electrical motion system.

\section{Experimental design and procedure}

A repeated-measures, within-participant experimental design was used, whereby all drivers experienced the same driving environment. Each data collection session lasted approximately 2.5 hours, which included a briefing session, a practice/familiarization drive, and four experimental drives, interspersed with a series of subjective data collection sessions, as outlined below. Upon arrival, each participant was provided with an experimental briefing, signed a consent form, and was given the opportunity to ask questions. Following a short practice drive ( $\approx 15$ minutes), the aim of which was to familiarize participants with vehicle controls and ensure they did not suffer any ill-effects from simulator exposure, they returned to the briefing area, for completion of the initial questionnaires (described below). Anyone who reported anything above slight simulator sickness was asked to discontinue driving, to avoid biasing the results. Participants then completed four simulator drives (separated by a short break). Exactly the same geometry was provided for each drive, but one included a set of oncoming vehicles whilst the other three did not. To ensure the driving task was as realistic as possible, and reduce variability across participants, drivers were encouraged to "drive as if you are in a hurry". At the end of the four drives, the participant completed another set of questionnaires, was thanked and provided $£ 30$ for their participation in the study.

\section{Development of scenarios with different risk levels}

Risk was presented through manipulations of the visual environmental scene and was varied by varying a number of factors: 'Road width', 'Road curvature radius and direction', 'Presence of oncoming traffic', 'Environment' (urban, rural), 'Contextual risk' (asphalt, grass, kerb, hedge, edge hatch, centre hatch, bus-stop, parked cars, work zone, pedestrian refuge), and 'Persistence of that contextual risk' $(250 \mathrm{~m}, 20 \mathrm{~m})$. Specifically, 'contextual risk' in this context defines the 
drivability of the road, based on the density, or static vs. dynamic nature, of the street furniture positioned along the road edge. Each of the four simulated road environments was constructed from 16 road segments $250 \mathrm{~m}$ in length, each with an associated level of risk, based on a combination of the above factors. Overall, participants repeated each level of risk five times, to facilitate multiple exposures to that risk. To simplify the presentation of results, 'Levels of contextual risk' were re-categorized as 'Low Risk', 'Static Risk', or 'Potentially Dynamic Risk', based on crash and comfort likelihood, taking perceptual cues of the road environment into account. 'Low risk' included segments with asphalt or grass at the road edge. 'Static Risk' included segments with a kerb, hedge, edge hatch, centre hatch, parked cars, or work zone. 'Potentially Dynamic Risk' included segments with a bus stop or pedestrian refuge, since these included pedestrians which could potentially move into the road.

\section{Dependent variables}

Various measures of lateral and longitudinal vehicle control were also recorded and analyzed, however, initial analysis revealed that only measures of speed correlated with SS scores. Therefore, the dependent variable used for statistical analysis in this study was the $95^{\text {th }}$ Percentile of Speed. Sensitive to individual differences, speed provides a key indicator of perceived risk, with speed typically reducing as perceived risk increases (Deery, 1999). To avoid variance in speed as drivers moved between segments, we only considered the middle portion of each road segment for the analysis, and only included segments where the risk persisted over the full $250 \mathrm{~m}$. Data were aggregated for each participant, across each repetition of the risk levels.

\section{Questionnaires}

To establish if driver characteristics were likely to influence risk propensity and driving style, a number of questionnaires were administered during various phases of the drive. These included the Arnett Inventory of Sensation Seeking (AISS; Arnett, 1994) questionnaire, used to determine an individual's sensation seeking, which is defined as a "need for novel and intense stimulation". 20 items are described, and participants are required to rate their response using 4-point Likert scale $(1=$ describes me very well to $4=$ does not describe me at all $)$. We also administered the Traffic Locus of Control (T-LOC; Özkan \& Lajunen, 2005) questionnaire, which measures the relationship between an individual's locus of control and their: risky driving, driving offences, and accidents. Participants used a 5-point scale to rate the frequency of occurrence of 17 listed behaviors. Finally, the 15-item Driver Style Questionnaire (DSQ; West, Elander, \& French, 1992), which measures attitudes to/engagement in different types of driving behavior was administered. Only AISS scores are reported in this paper, as our analysis showed there was no consistent relationship between T-LOC or DSQ scores and speed choice, for the different factors under investigation. AISS contains sub-scales of Intensity and Novelty, however, since there were no differences between the two, in terms of predicting speed choice, they were combined.

\section{RESULTS AND DISCUSSION}

Preliminary analysis showed that 'Environment' (urban/rural) and 'Levels of contextual risk' were the main factors that mediated the relationships between SS and speed choice and will, therefore, be used in the analysis. The remaining factors of 'Presence of oncoming vehicles', 'Road curvature', 'Lane width', and 'Persistence of contextual risk' were aggregated and form part of the means of 'Environment', 'Levels of contextual risk'. Figure 1 shows screenshots of example driving scenes, depicting the combination of levels 'Environment', 'Levels of contextual risk', 

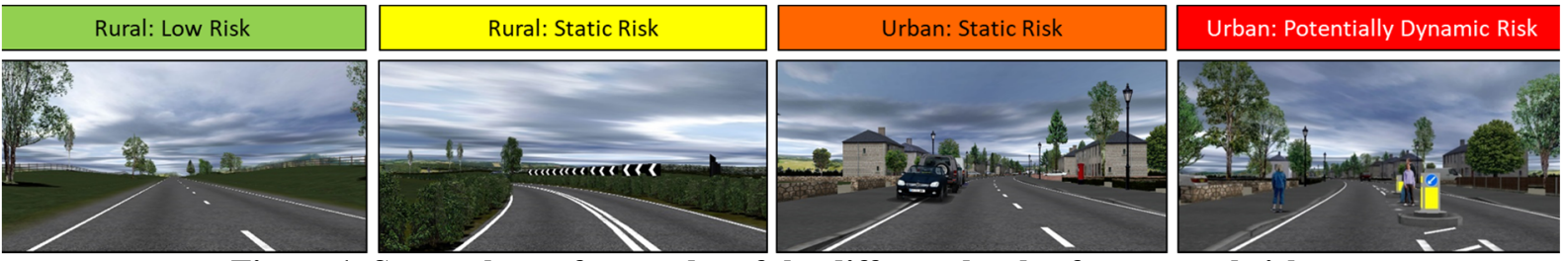

Figure 1. Screenshots of examples of the different levels of contextual risk

where the perceived risk was hypothesized to increase from left to right. To consider the relationship between SS and the $95^{\text {th }}$ percentile of speed across all risk levels, Pearson productmoment correlation coefficient was computed for all data. First, the overall relationship between SS and speed choice is considered, followed by a comparison between age groups, gender, road environment, and levels of contextual risks.

In line with previous research (e.g. Jonah, 1997; Dahlen et al., 2005), our data showed that, overall, there was a significant positive correlation between the two variables $(\mathrm{r}=.63, \mathrm{p}<.001)$, where increases in SS scores were correlated with increases in speed choice (Figure 2). In terms of age, Figure $3 \mathrm{~A}$ shows that there was a significant positive correlation between SS and the $95^{\text {th }}$ percentile of speed choice for drivers aged $<25$ years

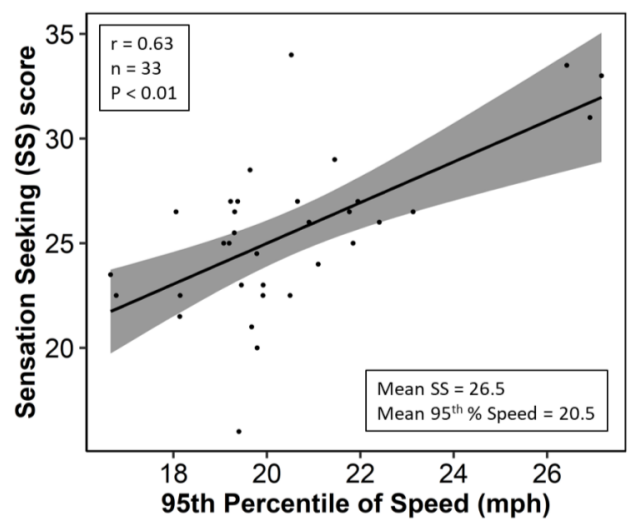

Figure 2. The relationship between SS and $95^{\text {th }} \%$ speed for all drivers in all contexts and $25-40$ years $(r=.84, p<.001$, and $r=.82, p<.001)$, respectively. There was no such correlation for drivers aged 40-60 years or over 60, who had lower average SS scores and speeding, compared to younger drivers. This pattern is not surprising, as SS is known to be higher in younger individuals (Zuckerman, 1971). Jonah (1997) suggests this may be because younger drivers are either more likely to accept risks while driving, to experience the thrill, or they may be misperceiving the risk because of their inexperience. For older drivers, on the other hand, experience and non-contextual decision-making skills (Gianfranchi et al., 2017) may suppress the tendency to act on sensation seeking in the interests of safety. In terms of gender, while males had higher SS scores ( $M=26.2$ vs $M=24.8$ for females), there was no difference in the relationship between SS and speed choice, with both males and females showing significant positive correlations $(\mathrm{r}=.69, \mathrm{p}<.005$, and $\mathrm{r}=.62, \mathrm{p}<.01)$, respectively. This is in contrast to Rimmö and Åberg (1999), who found a high correlation between SS scores and violations for male drivers. Although, caveats may exist for their questionnaire-based study, as it relied on selfreported record of violations.

A one-way repeated-measures ANOVA showed that there was a main effect of risk level (Rural low risk; Rural static risk, Urban static risk, Urban potentially dynamic risk) on speed choice $\left(\mathrm{F}(9,93)=62.141, \mathrm{p}<.001, \eta_{\mathrm{p}}{ }^{2}=.669\right)$, where drivers adopted slower speeds when navigating higher risk environments. Figure 3B shows that there was a significant positive relationship between SS and speed choice for each of the risk levels, but that the strength of the relationship weakened with increasing risk. Further analysis revealed that, while there was no difference between genders, there were some differences between age groups. The strength of the relationship held for the two younger age groups in all contexts. However, for the 40-50 and Over 60 groups, the association between SS and speed choice only existed in rural environments, while there was either no association, or a negative association in urban environments. This 
accounts for the weaker relationship at higher levels of risk. This also suggests that the tendency to speed for high-SS people was mediated, not only by the road environment, but also the level of contextual risk within each environment, and the age of the driver.
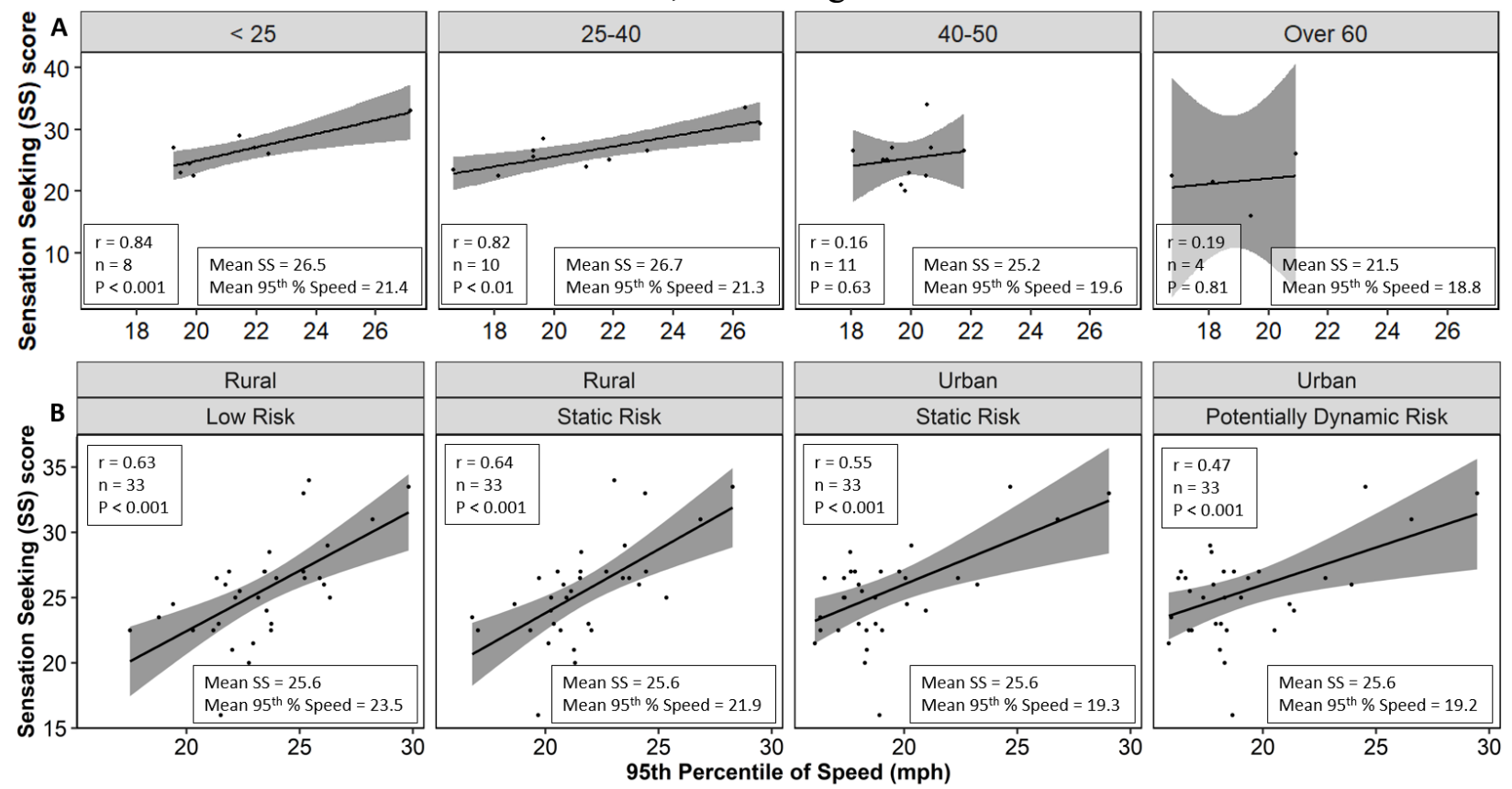

Figure 3. The relationship between AISS and $95^{\text {th }}$ Percentile of speed for A) different age groups and B) for different levels of contextual risk in the different road environments

\section{CONCLUSION}

This study investigated the relationship between drivers' sensation seeking propensity, and their speed choice in different road environments, which had different levels of contextual risk. Our results showed that, overall, there was a strong relationship between SS levels and speed choice, with higher sensation seekers tending to speed more. This was particularly the case for drivers under 40 years old. SS was also found to correlate positively across different levels of risk, however, the effect was more pronounced in road environments that were classified as less risky. Therefore, SS had the largest effect in low-risk contexts, and as the risk level rose, the effect of SS diminished. The speed calming effect of factors incorporated in more risky environments, coupled with the age, experience, and lower SS scores of drivers over 40 years old, tended to weaken the relationship between SS and speed choice. This shows that SS individuals may equalize risk or demand across situations more than others, but that in high-risk situations SS and non-SS people behave similarly. Although this study has provided a very rich set of driving environments for testing, there are some limitations. Challenges with nausea and simulator sickness prohibited the use of a large and equally sized sample for all age groups and thus the range of SS scores was relatively small. Therefore, the trends observed should be confirmed with research using a larger sample, more equally distributed between age groups. Regarding relevance to research on AVs, this work is the first step towards the HumanDrive project's goal to understand whether different AV driving styles are relevant for the ultimate acceptance of these vehicles by different sections of society.

\section{ACKNOWLEDGEMENTS}

This work is supported by the HumanDrive project, funded by the UK's Centre for Connected 
and Automated Vehicles (CCAV) and Innovate UK (Grant number TS/P012035/1). The authors gratefully acknowledge the contribution of Andrew Tomlinson, Anthony Horrobin, and Michael Daly for preparation of the simulated driving environment and of the data for analysis.

\section{REFERENCES}

Arnett, J. (1994). Sensation seeking: A new conceptualization and a new scale. Personality and Individual Differences, 16(2), 289-296.

Dahlen, E. R., Martin, R. C., Ragan, K., \& Kuhlman, M. M. (2005). Driving anger, sensation seeking, impulsiveness, and boredom proneness in the prediction of unsafe driving. Accident Analysis \& Prevention, 37(2), 341-348.

Deery, H.A. (1999). Hazard and Risk Perception among Young Novice Drivers. Journal of Safety Research, 30(4), 225-236.

Duffy, B. R. (2003). Anthropomorphism and the social robot. Robotics and autonomous systems, 42(3-4), 177-190.

Gianfranchi, E., Tagliabue, M., Spoto, A., \& Vidotto, G. (2017). Sensation Seeking, Non-contextual Decision Making, and Driving Abilities As Measured through a Moped Simulator. Frontiers in psychology, 8, 2126.

Hajiseyedjavadi, F., Louw T., Jamson, H., Merat., N., Li., P., Boer., E.R., Romano., R. (2019), Road factors and their impact on drivers' speed and lateral position control behavior. In Proceedings of the Tenth International Driving Symposium on Human Factors in Driver Assessment, Training and Vehicle Design, June 24-27, 2019, Santa Fe, New Mexico.

Hartwich, F., Beggiato, M., \& Krems, J. F. (2018). Driving comfort, enjoyment and acceptance of automated driving-effects of drivers' age and driving style familiarity. Ergonomics, 1-16.

Jonah, B. A. (1997). Sensation seeking and risky driving: a review and synthesis of the literature. Accident Analysis \& Prevention, 29(5), 651-665.

Loughran, D. S., \& Seabury, S. A. (2007). Estimating the Accident Risk of Older Drivers (Vol. 450). Rand Corporation.

Mirman, J. H., \& Kay, J. (2012). From passengers to drivers: Parent perceptions about how adolescents learn to drive. Journal of Adolescent Research, 27(3), 401-424.

Özkan, T., \& Lajunen, T. (2005). Multidimensional Traffic Locus of Control Scale (T-LOC): factor structure and relationship to risky driving. Personality and individual differences, 38(3), 533-545.

Rimmö, P. A., \& Åberg, L. (1999). On the distinction between violations and errors: sensation seeking associations. Transportation Research Part F: Traffic Psychology and Behavior, 2(3), 151-166.

Rudin-Brown, C. M., Parker, H. A., \& Malisia, A. R. (2003, October). Behavioral adaptation to adaptive cruise control. In Proceedings of the Human Factors and Ergonomics Society Annual Meeting, 47 (16); 1850-1854. Sage CA: Los Angeles, CA: SAGE Publications.

World Health Organization (2006). Road safety training manual Unit 2. Available: http://www.who.int/violence_injury_prevention/road_traffic/activities/roadsafety_training_manual_u nit_2.pdf.

West, R., Elander, J., \& French, D. (1992). Decision making, personality and driving style as correlates of individual accident risk. TRL Contractor Report (CR 309).

Zuckerman, M. (1991). Psychobiology of personality (Vol. 10). Cambridge University Press. 